\title{
Rotational vibration absorber for the mitigation of rail rutting corrugation
}

\author{
Christophe Collette*, Mihaita Horodinca and Andre Preumont \\ Department of Mechanical Engineering and Robotics, University of Brussels, Brussels, Belgium
}

(Received 6 February 2007; final version received 22 June 2008)

\begin{abstract}
Torsional vibrations of metro wheel sets are known to be involved in the wavelength-fixing mechanism of the rutting-type rail corrugation. In the first part of this paper, the basic conditions for this type of wear to appear are established using a theory developed in the frequency domain. The efficiency of a dynamic vibration absorber tuned to the first torsional resonance of the wheel set to mitigate rutting corrugation is evaluated numerically. In the second part, the phenomenon is studied on a quarter-scale test bench. The scaling laws for wheel-roller wear predictions are established. The efficiency of the dynamic vibration absorber is evaluated on the scaled bench. The results are compared with theoretical predictions from a linear model. Additionally, the measurements are compared with numerical results from a multi-body model portraying the experiment.
\end{abstract}

Keywords: rutting corrugation; dynamic vibration absorber; scaled test bench; multi-body model

\section{Introduction}

Under specific operating conditions, wheel-rail creep forces excite torsional vibrations of metro wheel sets responsible for a roll-slip phenomenon between the wheel and the rail. These oscillations are prone to develop a wavy wear of the railhead surface, known as rail rutting corrugation [1].

This type of corrugation has been observed in subway curves or where the vehicle accelerates and brakes [1-3]. The damage mechanism of this type of corrugation is a periodic wear of the rail surface due to periodic variations of the longitudinal creepage of the wheel. Main characteristics and a picture of this type of corrugation can be found in [1].

In a recent literature review, it has been mentioned that ' . . . there are good grounds to believe that rail rutting corrugation is the principal wavelength-fixing mechanism for corrugation in general on metro railways' [4]. The use of a dynamic vibration absorber (DVA) to alleviate rutting corrugation was suggested in [5], but, as far as the authors are aware, it has never been tested. The demonstration of the efficiency of the DVA is the main objective of this paper.

The paper is organised as follows. Section 2 describes the basic conditions for rutting corrugation to appear on the rail surface. Section 3 summarises Den Hartog's 'Equal peak'

*Corresponding author. Email: christophe.collette@ulb.ac.be

ISSN 0042-3114 print/ISSN 1744-5159 online

(C) 2008 Taylor \& Francis

DOI: $10.1080 / 00423110802339792$

http://www.informaworld.com 
design procedure and applies it to a torsional DVA. In Section 4, the effect of a DVA on theoretical corrugation growth coefficients is established. Finally, in Section 5, an experiment reproducing typical operating conditions in which rutting corrugation appears is considered. The experimental set-up is a quarter-scale test bench from the New Technologies Laboratory (INRETS-France) [6]. Scaling laws for wheel-roller wear predictions are established. The numerical efficiency of the DVA in mitigating rail rutting corrugation is evaluated in terms of the frictional power dissipated in the contact patch. Measurements are compared with numerical results from a multi-body model of the test bench which is portraying the experiment.

\section{Rail rutting corrugation}

\subsection{Background}

Torsional vibrations of railway wheel sets are thought to be involved in corrugation since nearly half a century. Actually, a torsional resonance of the wheel set can be involved in a corrugation mechanism only when the torsional vibrations are sustained by a variation of the vertical contact force and/or a decreasing slope of the friction curve [1]. For instance, corrugation of this type can be initiated by a joint between two rails. However, as the contact between the wheel and the rail behaves like a big damper in the longitudinal direction, the corrugation fades out after a few oscillations if the torsional vibration is not sustained as mentioned above. A mathematical model was developed in [7] to describe rail corrugation as a wear process resulting from the combination of torsional vibrations of the driving wheels and longitudinal vibrations of the rail. Using their simple model, they were additionally able to derive an analytical formulation for the frictional power. A model in which torsional vibrations of the wheel set are the main causes for rail corrugation was presented in [8]. This type of corrugation was definitively classified as rail 'rutting corrugation' in [1], due to its similarities with corrugated dirt roads. More recently, the formation mechanism of rutting corrugation has been studied experimentally on a full scale roller rig in $[9,10]$. In this case, torsional oscillations of the wheel set are combined with high vertical contact forces arising from the unsprung mass bouncing on the support stiffness. This wavelength-fixing mechanism is explained in more detail in the next section.

\subsection{Wavelength-fixing mechanism}

Wheel and rail profiles impose the railway vehicle to follow the track. This constrained motion induces high wheel-rail contact creep forces, and stresses in the vehicle-track system. The type of stress depends on the geometry of the system and the operating conditions. In this paper, only torsional stresses induced in the wheel set are considered. There are a number of phenomena responsible for this type of stresses, including the difference in rail length of curved track sections, the wear of wheel and rail profiles, a bad adaptation of the vehicle speed to the track superelevation and driving torques applied on the gear box. If there is no variation in the vertical contact force, the stress accumulated in the wheel set is relaxed, above the sticking limit, by a constant slip of the wheel on the rail.

On the other hand, during the passage of the vehicle, the surface roughness imposes a relative displacement between the wheel and the rail at each contact point, which creates the vertical dynamic contact forces. In this case, the stress accumulated in the wheel set is relaxed at the frequencies of high variations in the vertical contact force (e.g. peaks in the contact force spectrum). If the frequency of a torsional resonance of the wheel set is the same as the 
frequency of a peak in the vertical force spectrum, the relaxation is amplified, giving birth to rutting corrugation. Classical examples of the peaks of the contact force are given hereafter.

Figure 1 shows the vertical receptance of a track with concrete sleepers, and how this receptance is changed when the unsprung masses of the vehicle are laid on the track. The frequency of the first mode is decreased, and an anti-resonance appears in addition to the anti-resonance already existing for the track alone. The model used to obtain these curves is presented in the Section 4.1.

Figure 2 shows the vertical contact force generated on the same system by the rail roughness. Peaks of this spectrum correspond to the highest oscillations of the vertical contact force, which impose the relaxation of the stress accumulated in the wheel set. The first peak corresponds to a bouncing of the unsprung masses of the vehicle on the track stiffness; the second peak corresponds to the sleeper resonance; the third corresponds to the contact resonance. Below this last resonance, the displacement between the wheel and the rail corresponds, to a large extent, to the rail irregularity; above it, the irregularity is substantially compensated by local deformations of the wheel and the rail.

In this case, rutting corrugation can appear if the frequency of a torsional resonance of the wheel set corresponds to the frequency of the bouncing of the unsprung masses of the vehicle. This is sometimes the case for the first torsional resonance of subway wheel sets [1]. Rutting corrugation can also appear if a torsional resonance of the wheel set corresponds to the vertical resonance of the sleeper. This is sometimes the case for the second torsional resonance of subway wheel sets and a track with concrete sleepers. The coincidence of a peak in the vertical contact force and a torsional resonance is amplifying the phenomenon of rutting corrugation, but is not a necessary condition for its appearance (see [5,11]). It can appear as soon as the torsional vibrations are sustained by a variation of the vertical contact force. When it exists, a decreasing slope of the friction curve can also contribute to sustain the torsional vibrations of the wheel set.

Corrugation usually appears on the rail where the dynamic load is high. For instance, in the case of a curved track, corrugation appears usually on the low (inner) rail as depicted in Figure 3.

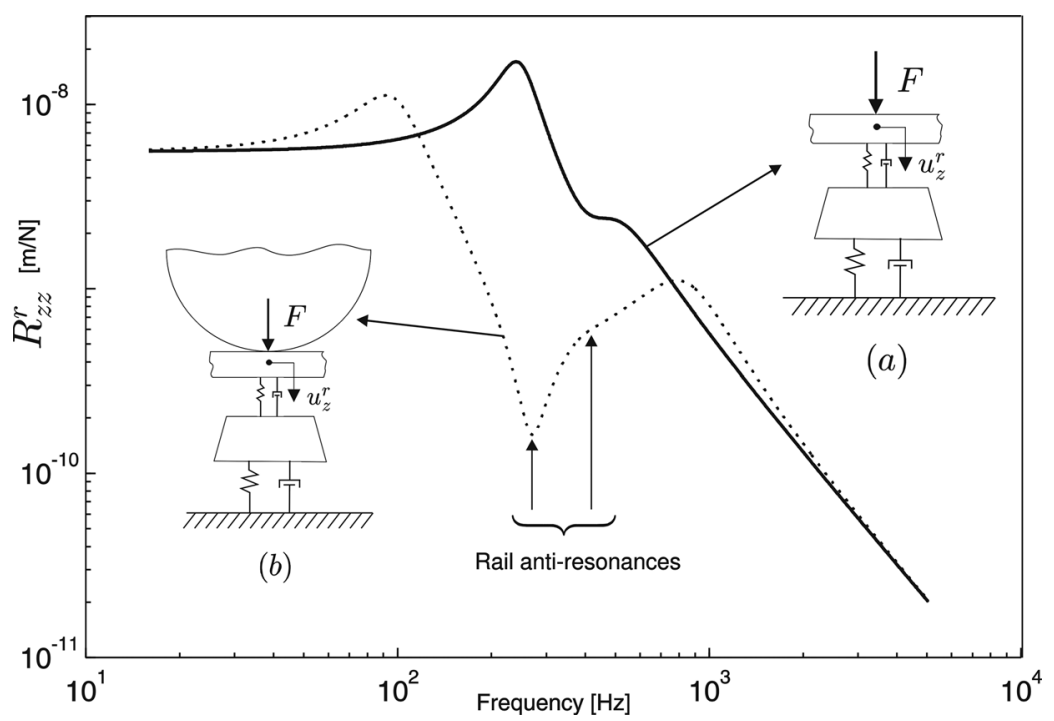

Figure 1. Typical vertical receptance of a track: (a) without unsprung mass (solid line); (b) with unsprung mass (dotted line). 


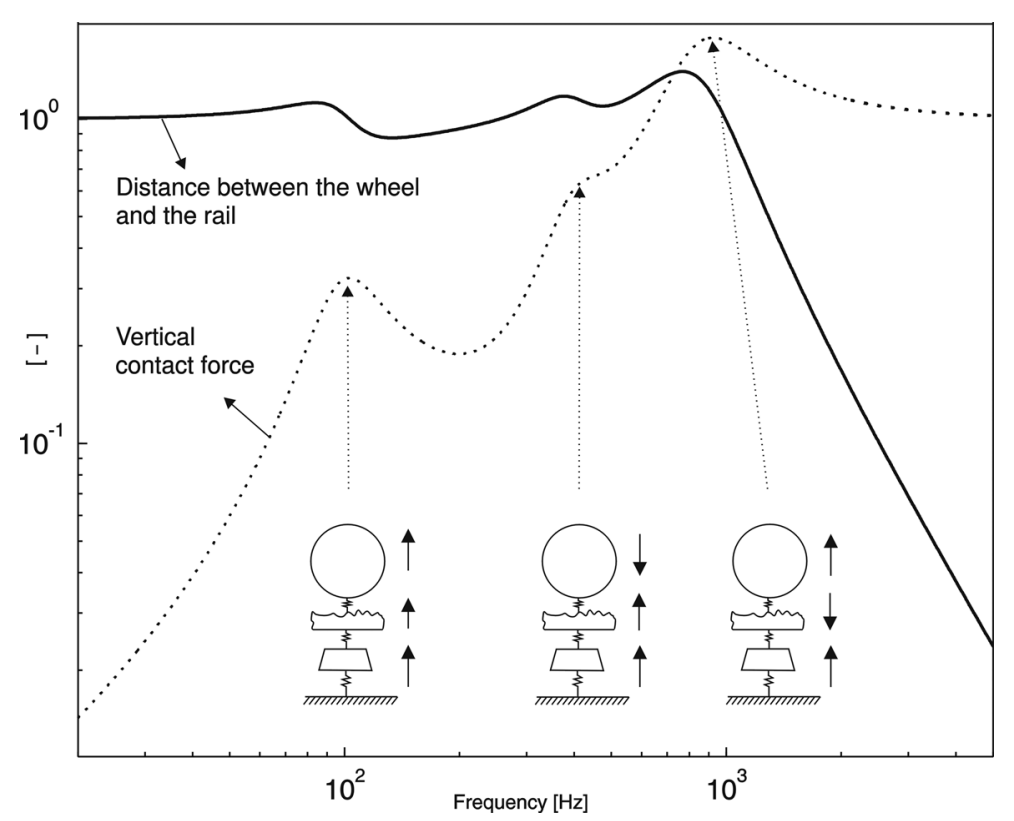

Figure 2. Vertical contact force $\left(N / k_{\mathrm{c}} z\right)$ and relative displacement between the wheel and the rail $(d / z)$.

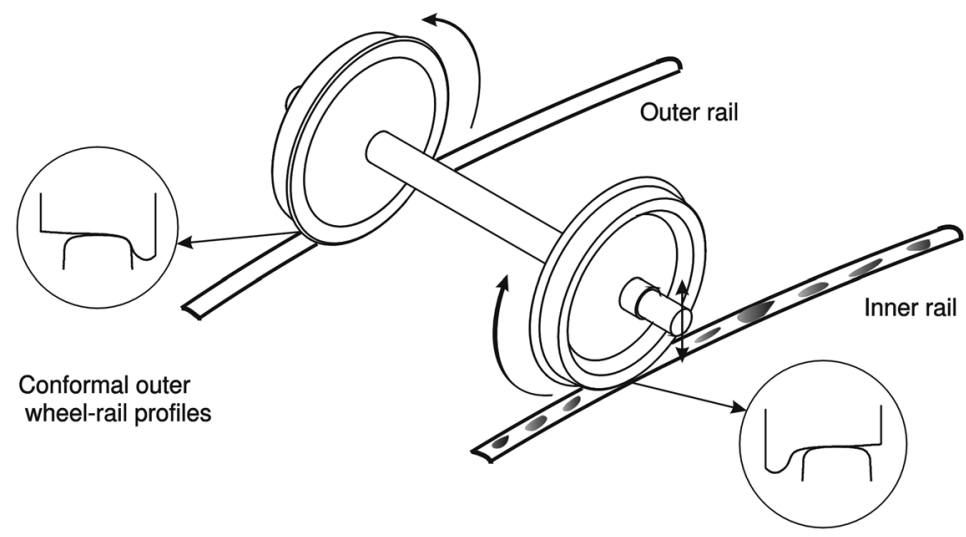

Figure 3. Rutting corrugation; corrugation appears most of the time on the inner rail because of the lower axle load and non-conformal contact.

\section{Torsional dynamic vibration absorber}

\subsection{Motivation}

Once the rails have been damaged, grinding is the only way of removing the existing rutting corrugation from the rail surface, but it does not prevent the phenomenon of reappearing after a while.

So far, effective measures to prevent rutting corrugation are of four types [1]: (i) decrease the vertical force (i.e. use vertically soft rail pad to decrease the vertical resonances [12-14]); (ii) use harder rail steel [1]; (iii) decrease the longitudinal creep force (improve the steering of the vehicle in curve using resilient yaw suspension or steering bogie, less severe curves on freight track [1]); (iv) modify the friction coefficient with lubricant [15] or friction modifier 
$[16,17]$. Solutions of the first three types are acting on the causes of the phenomenon, but need some modifications of the existing vehicle-track system. Solutions of the fourth type have proved their efficiency but have other drawbacks. The solution developed in this work is based on the use of a DVA to reduce the vibration amplitude of the torsional mode of the wheel set. This idea was originally suggested in [5], but has not been tested yet, as far as we know. DVAs have already been applied successfully on wheels and rails, for creep-related disturbances, especially in the high-frequency range, above $500 \mathrm{~Hz}$, for noise reduction [18] and alleviation of corrugation induced by the so-called rail pinned-pinned resonances [19].

\subsection{Design procedure}

The DVA was introduced by Den Hartog in 1929 [20]; it consists of a small spring-mass system tuned in such a way as to dissipate the vibratory energy of the targeted mode. It can be applied to translational as well as to rotational degrees of freedom (d.o.f.). The procedure for the design of a DVA for a single d.o.f. system is widely available in the literature (e.g. [21,22]). For a multi d.o.f. system with well-separated modes, Figure 4a, the design procedure is essentially the same. When the tuned DVA is attached to the structure, the behaviour in the vicinity of the targeted mode as seen from the attachment point, Figure 4b, is essentially the same as that of a single d.o.f. oscillator and the classical design procedure can be applied (e.g. [23]). The DVA has three design parameters: (i) the mass (inertia) ratio $\lambda=m_{a} / m$ which expresses the ratio between the mass of the absorber and that of the system seen from the attachment point, (ii) the frequency ratio which expresses the ratio between the natural frequency of the DVA (when the attachment point is fixed) and that of the targeted mode in the original system (Figure 4a) and (iii) the damping coefficient.

The extension of the DVA from translational d.o.f. to rotary d.o.f is straightforward, and the only special aspect is the inertia associated with the targeted mode. In this study, it is taken according to $m=\mu_{k} / \phi^{2}(d)$, where $\mu_{k}$ is the generalised mass of the targeted mode and $\phi_{k}(d)$ is the modal amplitude at the location of the DVA.

The inertia ratio $\lambda$ is first selected by the designer; it is typically of a few percent of the original mass of the system (in the example presented below, $\lambda=0.036$ for the first torsional mode, and $\lambda=0.027$ for the second torsional mode). For a given attachment point, the efficiency of the DVA increases with $\lambda$. The selection of the damper stiffness is now examined. The direct receptance of a lightly damped single d.o.f. system with a tuned DVA is represented in Figure 5a. It consists of two peaks, the amplitudes of which go up and down as a function of the damping ratio. It turns out that for a given value of the frequency ratio $\beta=\omega_{a} / \omega$ (i.e. a given value of the stiffness $k_{a}$ ), all the curves intersect at the two points $\mathrm{P}$ and Q shown in Figure 5b. According to the so-called equal peak design procedure, the DVA

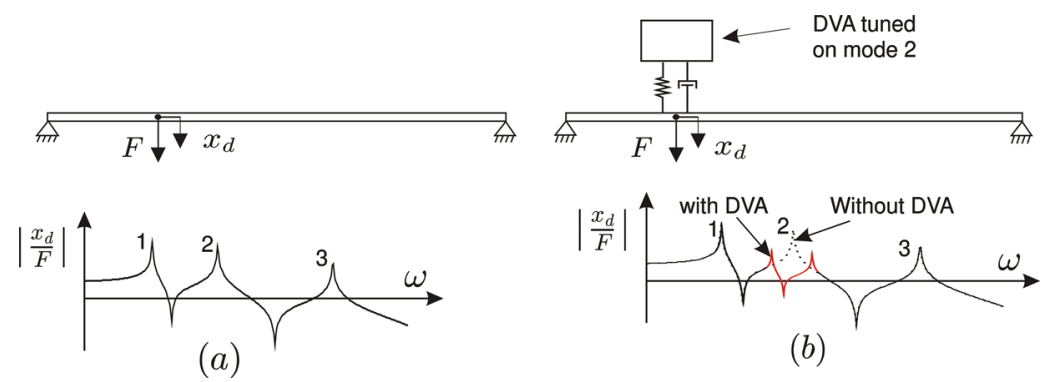

Figure 4. Effect of a dynamic vibration absorber on mode 2 of a typical structure. 


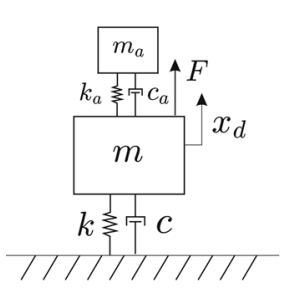

(a)

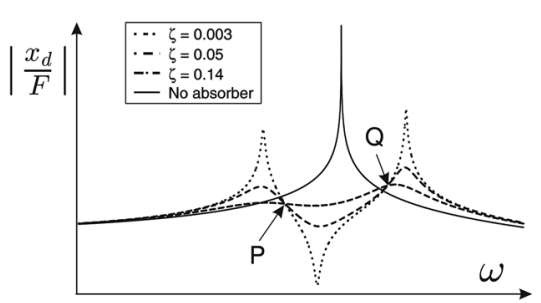

(b)

Figure 5. Equivalent structure in the frequency range of the targeted resonance.

stiffness is selected in such a way that the two points $\mathrm{P}$ and $\mathrm{Q}$ have equal amplitude. This is achieved if

$$
\beta=\frac{\omega_{a}}{\omega}=\sqrt{\frac{k_{a}}{m_{a}} \frac{m}{k}}=\frac{1}{1+\lambda}
$$

Once the frequency ratio has been selected to achieve the same amplitude for $\mathrm{P}$ and $\mathrm{Q}$, the damping coefficient $\zeta=c_{a} /\left(2 \omega_{a} m_{a}\right)$ can be selected so as to minimise the maximum amplitude of the receptance function (see Figure 5b); this is sometimes referred to as $H_{\infty}$ optimisation. This is achieved when the two peaks lie exactly on $\mathrm{P}$ and $\mathrm{Q}$. The corresponding value of the damping ratio of the DVA is

$$
\zeta_{\mathrm{opt}}=\sqrt{\frac{3 \lambda}{8(1+\lambda)}}
$$

\subsection{Mechanical arrangement}

The DVA should be located where the modal amplitude of the targeted mode is maximum. For the first torsion mode of the wheel set (Figure 6a), it should be as close as possible to the wheel (actually, a DVA design directly mounted on the wheel has been proposed [24]); for the second mode, it can be mounted either close to one of the wheels, or close to the disk brake (Figure $6 b$ ), depending on the space available along the axle. Figure $6 \mathrm{c}$ shows a complete wheel set on which are mounted the two DVAs. The mechanical arrangement consists of two metallic half-rings (inertia) fixed to the axle with a thin layer of viscoelastic material (providing the stiffness and damping). ${ }^{1}$ The system is extremely simple, maintenance-free and can be mounted on the axle without any modification of the wheel set. In this study, the viscoelastic material has been assumed to behave according to constant stiffness and viscosity in the vicinity of the first mode (Kelvin-Voigt model).

\subsection{Application to the LTN-INRETS test bench}

The foregoing methodology has been followed to design a DVA for the first and second torsional modes of a quarter-scale metro wheel set of the test bench from the LTN-INRETS. The rotational inertia ratio $(\lambda)$ is 0.036 for the first mode and 0.027 for the second mode; the optimum DVA parameters obtained by the design procedure described above are given in Table 1. It was not possible to obtain exactly the optimum values of $k_{a}$ and $c_{a}$ because the stiffness and damping properties of viscoelastic materials cannot be chosen independently. The actual values as obtained by identification from experimental data are also shown in Table 1; the damping coefficient is 2.4 times larger than the optimum value. Figure 7 shows the calculated 

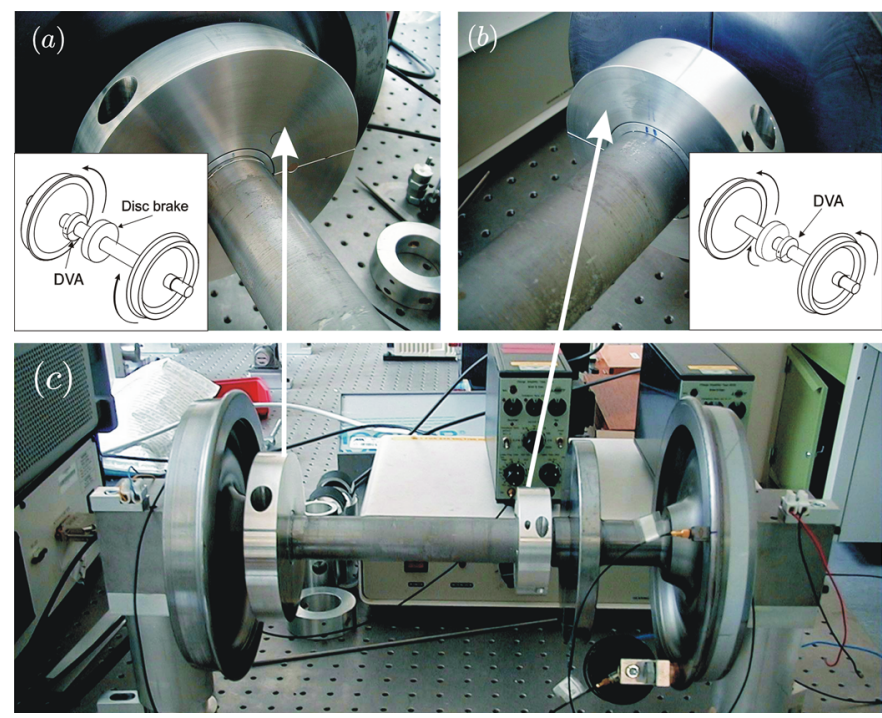

Figure 6. DVA mounted on the wheel set axle: (a) tuned on the first torsional resonance; (b) tuned on the second torsional resonance; (c) complete wheel set on which are mounted the two DVAs.

Table 1. Characteristics and amplitude attenuation of the dynamic vibration absorber.

\begin{tabular}{lcccc}
\hline & $k_{a}(\mathrm{kNm} / \mathrm{rad})$ & $c_{a}(\mathrm{Nms} / \mathrm{rad})$ & Mode 1 $(\mathrm{dB})$ & Mode 2 $(\mathrm{dB})$ \\
\hline Optimum (Den Hartog) & 4.1405 & 0.6647 & 23 & 19 \\
Measurement & 3.4124 & 1.748 & 18 & 17 \\
\hline
\end{tabular}

and measured direct receptance of the wheel set in the longitudinal (rolling) direction, at an extremity of the wheel. Figure 7 a shows the peak corresponding to the first torsional resonance and Figure $7 \mathrm{~b}$ shows the peak corresponding to the second torsional resonance. The four curves are, respectively, (i) the measured receptance without DVA, (ii) the calculated receptance without DVA, (iii) the receptance with DVA calculated for the optimum value of the parameters (first line of Table 1) and (iv) the receptance with DVA measured with the actual value of the parameters (second line of Table 1). One sees that, because of the larger damping coefficient used in the experiment, a single peak appears in the experimental receptance with DVA; in spite of this, an attenuation of $18 \mathrm{~dB}$ (resp. $17 \mathrm{~dB}$ ) is achieved for the first (resp. second) mode, instead of $23 \mathrm{~dB}$ (resp. $19 \mathrm{~dB}$ ) for the optimum parameters. This confirms that the DVA optimum design is robust with respect to the variation of its parameters. This aspect is further confirmed below.

\subsection{DVA sensitivity}

The optimum values of the stiffness and damping parameters are given in Equations (1) and (2). Any departure from these optimal values entails a reduction in the DVA performance. Mistuning can result from statistical variations in the wheel set natural frequency, the mounting of the DVA, as well as environmental conditions (temperature, humidity, ageing, etc.). Figure 8 shows the attenuation of the longitudinal receptance measured at the frequency of the first torsional resonance, using a DVA whose rotary inertia is changed with respect to the optimal value. One sees that an attenuation of $15 \mathrm{~dB}$ is maintained for a wide range of variation in the moment of inertia. 

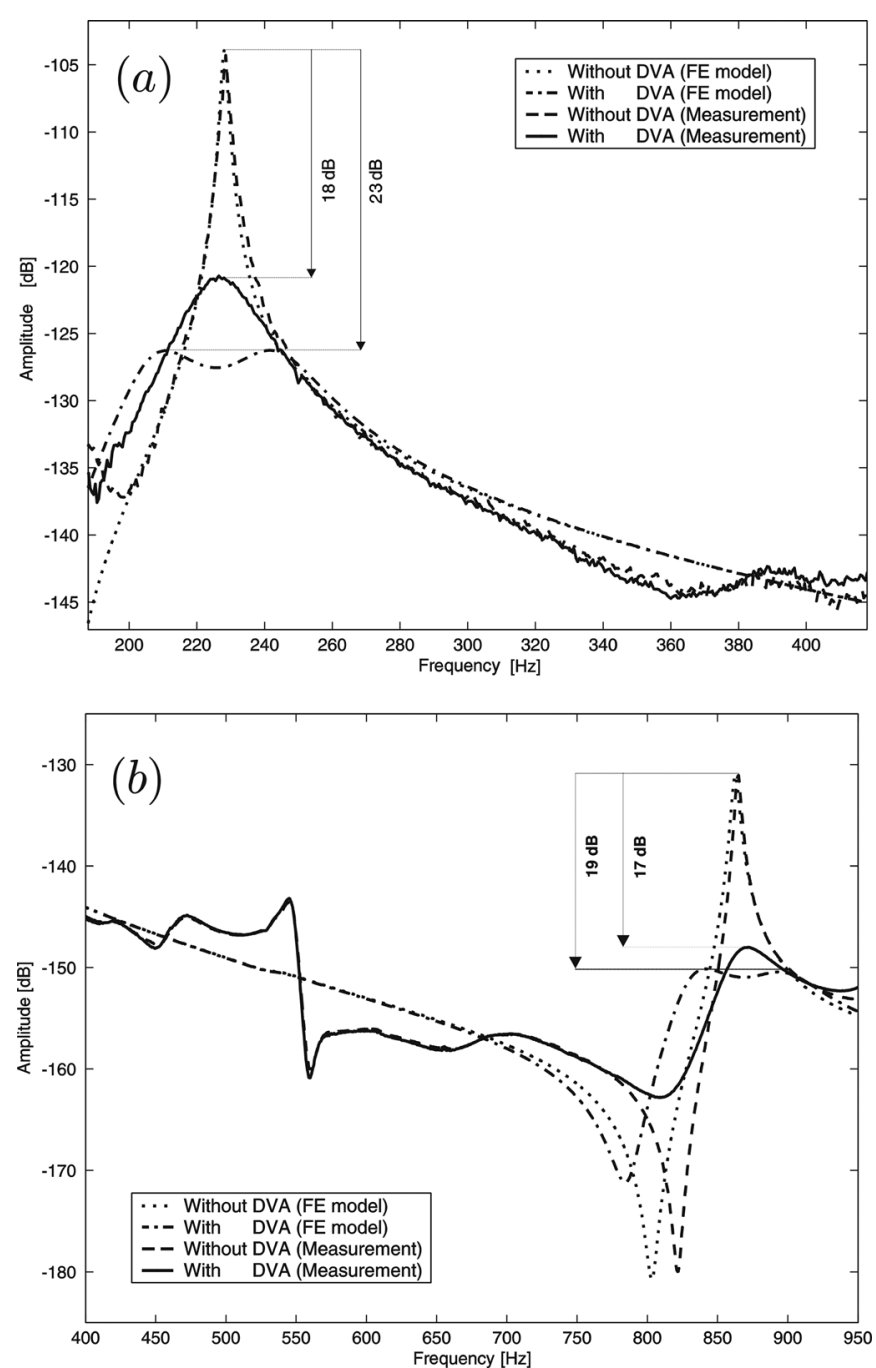

Figure 7. Longitudinal (in the rolling direction) receptance of the wheel set wheel: FE model without DVA (dotted line); FE model with DVA (dashed dotted line); measurement without DVA (dashed line); measurement with DVA (solid line). (a) First torsional resonance; (b) second torsional resonance.

\section{Effect of the DVA on corrugation growth}

This section analyses the influence of the DVA on the corrugation growth coefficients derived from the equations of the vehicle-track dynamics.

\subsection{Model of the vehicle-track dynamics}

The vertical dynamics of the wheel-rail contact is governed by Hertz's theory, giving a nonlinear relationship between the load and the deformation of the bodies in contact (Figure 9a). For each load, this relationship can be linearised as depicted in Figure $9 \mathrm{~b}$ and

$$
\Delta N=k_{\mathrm{c}} \Delta d
$$




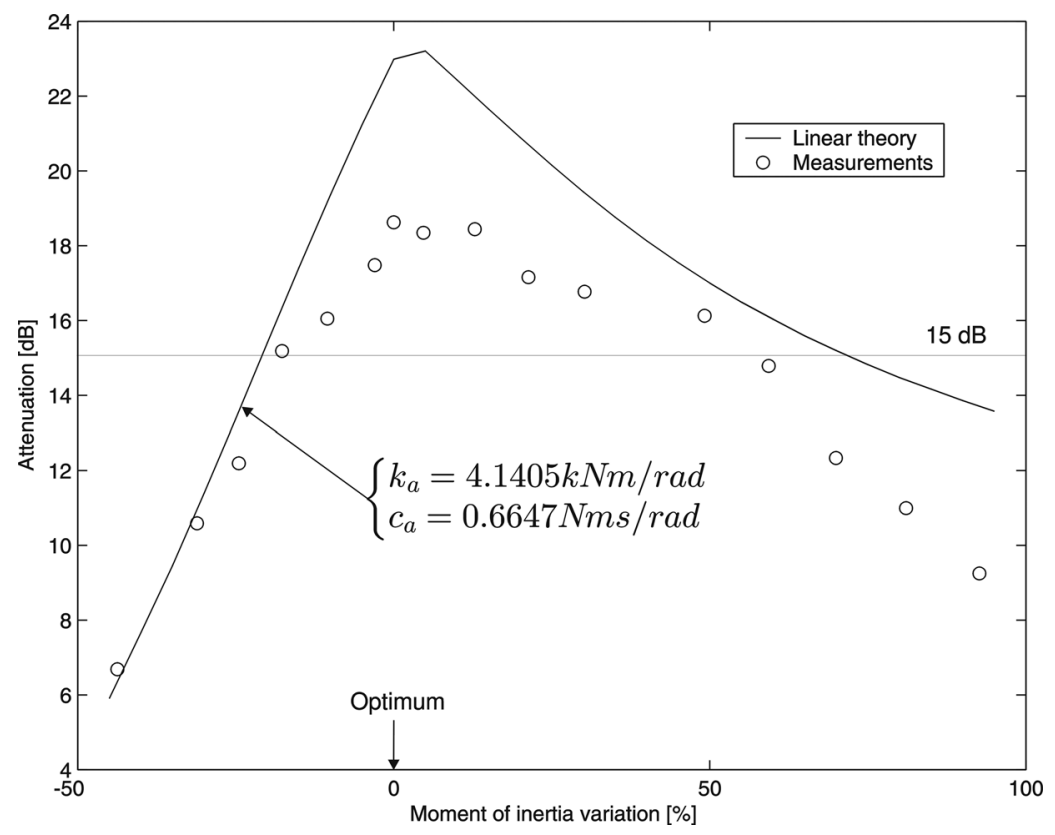

Figure 8. Efficiency of the DVA in non-tuned conditions.

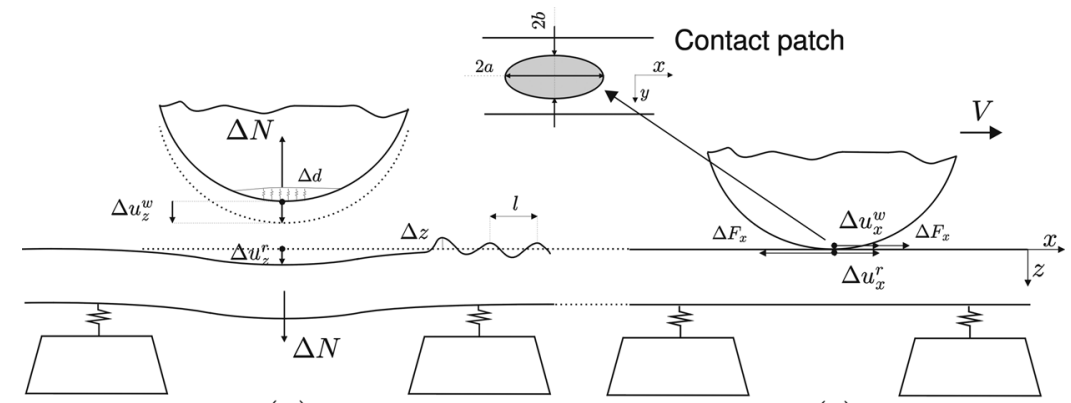

(a)

(c)

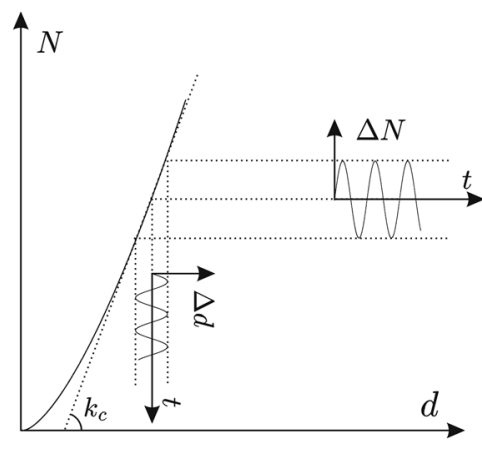

(b)

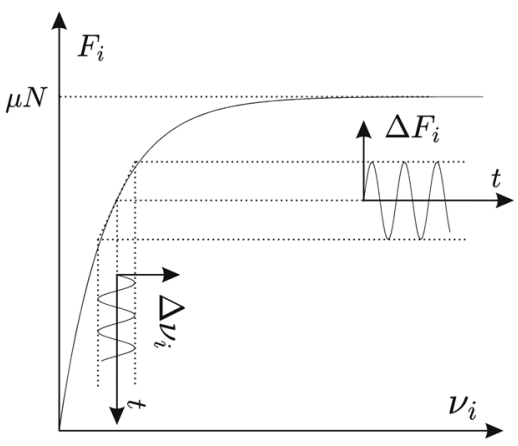

(d)

Figure 9. (a) Vertical vehicle-track dynamics; (b) linearisation of the vertical contact law; (c) longitudinal vehicle-track dynamics; (d) linearisation of the longitudinal creepage force $v s$ creepage law. 
where $\Delta d$ is the variation of the distance between the wheel and the rail induced by a variation of the vertical force $\Delta N$ and $k_{\mathrm{c}}$ is called the contact stiffness. Taking the vehicle and track dynamics into account, an irregularity of the rail surface $\Delta z$ leads to a combination of a wheel displacement $\Delta u_{z}^{\mathrm{w}}$, a rail displacement $\Delta u_{z}^{\mathrm{r}}$, and a deformation at the wheel-rail interface $\Delta d$ :

$$
\Delta z=\Delta d+\Delta u_{z}
$$

where $\Delta u_{z}=\Delta u_{z}^{\mathrm{w}}-\Delta u_{z}^{\mathrm{r}}$. The vertical displacements of the wheel and the rail are given by

$$
\begin{aligned}
\Delta u_{z}^{\mathrm{w}} & =R_{z z}^{\mathrm{w}} \Delta N \\
\Delta u_{z}^{\mathrm{r}} & =-R_{z z}^{\mathrm{r}} \Delta N
\end{aligned}
$$

where $R_{z z}^{\mathrm{w}}$ and $R_{z z}^{\mathrm{r}}$ are, respectively, the vertical direct receptances of the vehicle and the track. The longitudinal creepage $v_{x}$ between the wheel and the rail is given by

$$
v_{x}=\frac{\dot{u}_{x}^{\mathrm{w}}-\dot{u}_{x}^{\mathrm{r}}}{V}
$$

where $\dot{u}_{x}^{\mathrm{w}}$ and $\dot{u}_{x}^{\mathrm{r}}$ are, respectively, the longitudinal displacements of the wheel and the rail, and $V$ the vehicle speed (Figure 9c). In the frequency domain, small linear variations in the longitudinal creepage are expressed by

$$
\Delta v_{x}=\frac{\mathrm{j} \omega \Delta u_{x}}{V}
$$

where $\Delta u_{x}=\Delta u_{x}^{\mathrm{w}}-\Delta u_{x}^{\mathrm{r}}$ and $\omega=2 \pi f=2 \pi V / l(f$ is the frequency and $l$ the wavelength of the irregularity).

The relationship between the longitudinal creepage and the associated creep force between the wheel and the rail is given by a non-linear phenomenological law shown in Figure 9d. Again, this law can be linearised as follows:

$$
\Delta F_{x}=\frac{\partial F_{x}}{\partial N} \Delta N+\frac{\partial F_{x}}{\partial v_{x}} \Delta v_{x}
$$

The longitudinal displacements of the vehicle and the track are expressed as

$$
\begin{aligned}
\Delta u_{x}^{\mathrm{w}} & =R_{x x}^{\mathrm{w}} \Delta F_{x} \\
\Delta u_{x}^{\mathrm{r}} & =-R_{x x}^{\mathrm{r}} \Delta F_{x}
\end{aligned}
$$

where $R_{x x}^{\mathrm{w}}$ and $R_{x x}^{\mathrm{r}}$ are, respectively, the longitudinal direct receptances of the vehicle and the track.

Summarising Equations (3) to (11), the vertical and longitudinal vehicle-track dynamics can be expressed by the following system of linear equations [25]:

$$
\left[\begin{array}{cccccc}
1 & 0 & 1 & 0 & 0 & 0 \\
-k_{\mathrm{c}} & 1 & 0 & 0 & 0 & 0 \\
0 & R_{z z}(\omega) & -1 & 0 & 0 & 0 \\
0 & 0 & 0 & 1 & 0 & R_{x x}(\omega) \\
0 & 0 & 0 & \mathrm{i} \omega / V & -1 & 0 \\
0 & \alpha & 0 & 0 & \beta & -1
\end{array}\right]\left[\begin{array}{c}
\Delta d \\
\Delta N \\
\Delta u_{z} \\
\Delta u_{x} \\
\Delta v_{x} \\
\Delta F_{x}
\end{array}\right]=\left[\begin{array}{l}
1 \\
0 \\
0 \\
0 \\
0 \\
0
\end{array}\right] \Delta z
$$

where $R_{z z}(\omega)=R_{z z}^{\mathrm{w}}(\omega)+R_{z z}^{\mathrm{r}}(\omega)$ and $R_{x x}(\omega)=R_{x x}^{\mathrm{w}}(\omega)+R_{x x}^{\mathrm{r}}(\omega)$ (see Figure 1$) ; \alpha=$ $\partial F_{x} / \partial N$ and $\beta=\partial F_{x} / \partial v_{x}$. The first three equations describe the vertical dynamics and the last three equations express the longitudinal dynamics. $\alpha$ and $\beta$ are obtained from the creepage-creep force phenomenological law; analytical formulae can be found in $[2,3]$ for the case of an exponential law. Contact filters are not considered in this work, because their contribution becomes significant only when the wavelength is very small $(l / a<3,[26])$. 


\subsection{Wear model}

After each passage of a wheel on the rail, the profile of the rail is slightly modified due to the wear generated by the contact between these two bodies. According to [27], the mass $\Delta m$ removed after the $n$th wheel passage is proportional to the work $\Delta W$ of contact friction forces

$$
\frac{\partial \Delta m(\omega, n)}{\partial n}=k_{0} \Delta W(\omega, n)
$$

with $k_{0}$ the proportionality coefficient. Equation (13) can be transformed into

$$
\frac{\partial \Delta z(\omega, n)}{\partial n}=-\frac{k_{0}}{2 \rho b V} \Delta P_{x}(\omega, n)
$$

where $\rho$ is the density, $2 b$ is the width of the Hertzian contact (Figure 9) and $\Delta P_{x}=$ $V\left(\Delta v_{x} F_{x}+v_{x} \Delta F_{x}\right)$ is the frictional power variation. Using Equation (12) to express $\Delta v_{x}$ and $\Delta F_{x}$ as functions of the system dynamics, Equation (14) can be written as

$$
\frac{\partial \Delta z(\omega, n)}{\partial n}=G(\omega) \Delta z(\omega, n)
$$

which governs the growth of the corrugation. The corrugation growth coefficient is given by the real part of $G(\omega)$

$$
\Re\{G(\omega)\}=-\frac{k_{0}}{2 \rho b V} \Re\left\{\frac{\alpha\left[V v_{x}-\mathrm{j} \omega F_{x} R_{x x}\right]}{\left[R_{z z}+1 / k_{c}\right]\left[1+\beta / V \mathrm{j} \omega R_{x x}\right]}\right\}
$$

Positive values of $\Re\{G(\omega)\}$ correspond to wavelengths that will be magnified in the roughness spectrum. Alternatively, negative values of $\Re\{G(\omega)\}$ correspond to wavelengths that will be flattened. The higher the value of $\Re\{G(\omega)\}$, the more rapidly corrugation will develop at the corresponding frequency.

\subsection{Effect of the DVA}

The effect of a DVA on the corrugation growth is shown in Figure 10 for the case of a vehicle rolling on a ballasted track (see the typical receptances in [2]). For the specific operating conditions described in Figure 10b, the contribution of the first torsional resonance of the wheel set to the corrugation growth coefficient has been divided by more than two when a DVA tuned to this mode is mounted on the wheel set axle.

\subsection{Parametric study}

For the specific case considered in Section 4.3, the evolution of the amplitude of the sinusoidal rail profile is shown in Figure 11a for various values of the inertia ratio $\lambda$. Without DVA $(\lambda=0)$, assuming that the rail surface must be ground ones the amplitude reaches a value of $0.8 \mathrm{~mm}$ peak to peak, the period elapsed between two grindings is about 9 months. When the DVA is mounted on the wheel set axle, this period increases as the inertia of the DVA increases (Figure 11b). For instance, an inertia ratio $(\lambda)$ of 0.02 can reduce the number of grindings by two, and the associated maintenance costs. 

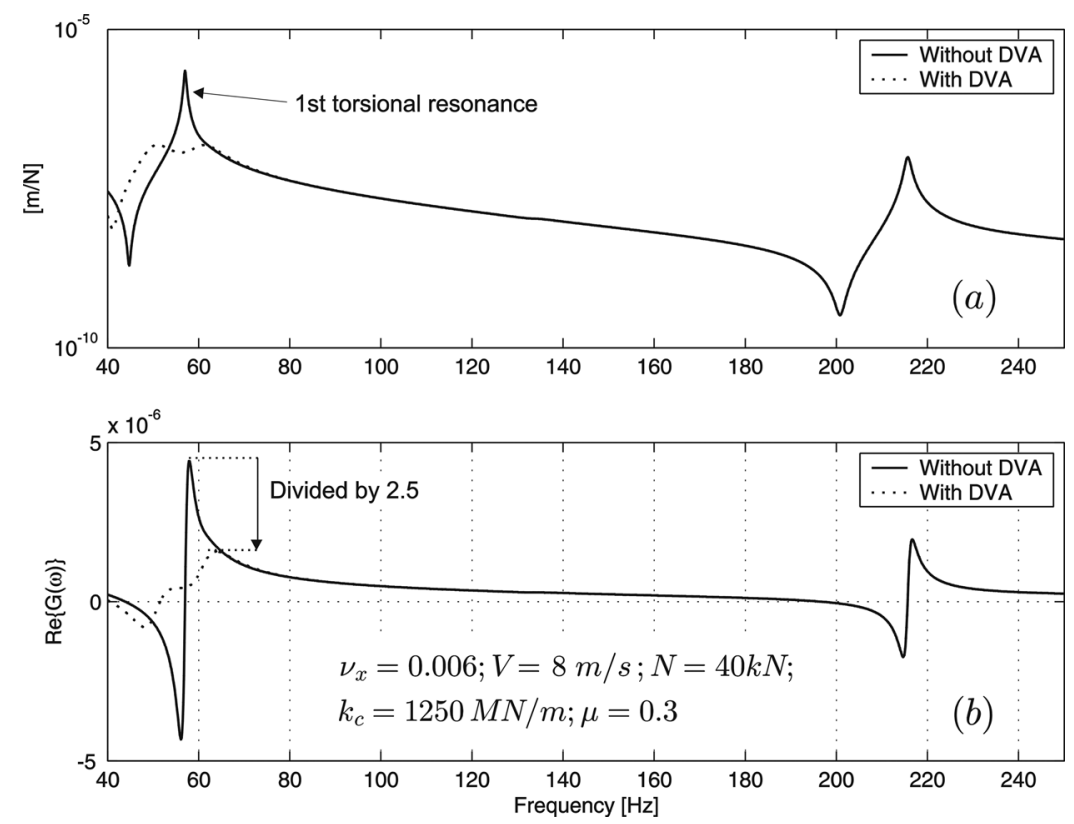

Figure 10. Effect of a DVA on the corrugation growth coefficient: (a) typical wheel set receptance in the longitudinal direction; (b) corrugation growth coefficient in the longitudinal direction.
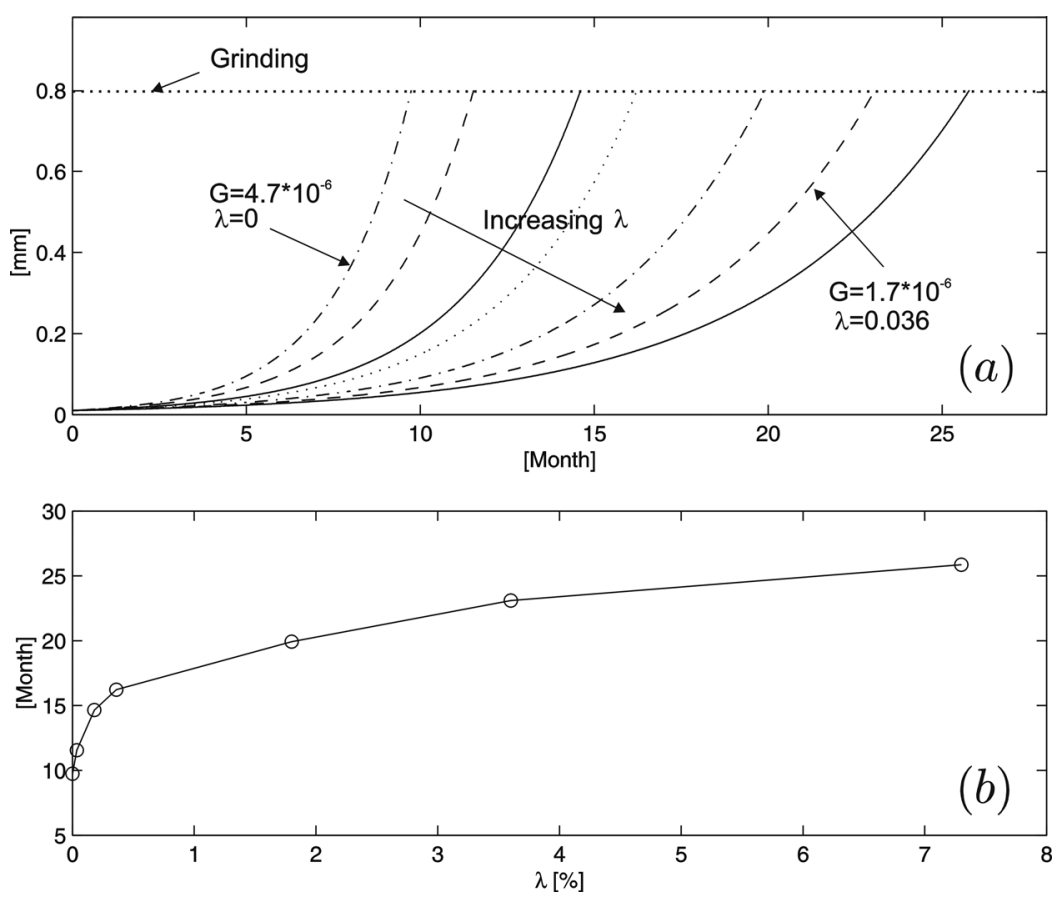

Figure 11. (a) Effect of the inertia ratio on the corrugation growth coefficent; (b) increase in the duration between two grindings for various inertia ratios. 


\section{Validation}

In the previous section we have pointed out the effect of a DVA on the corrugation growth coefficient related to rutting corrugation. In order to verify experimentally this relation, this section studies the phenomenon of rutting corrugation using a test bench from the New Technologies Laboratory (INRETS-France) [6]; the test set-up has been properly adapted for the purpose of this study. First, the laws for the prediction of wheel-roller wear on the scaled test bench is established. Then, an experiment reproducing typical operating conditions in which the mechanism of rutting corrugation appears is considered, and the results are compared with theoretical predictions. Measurements are compared with numerical results from a multi-body model of the test bench which is portraying the experiments.

\subsection{Scaling laws}

Depending on the phenomenon, different scaling strategies have been used for the design of scaled test benches [28]. The aim of INRETS was to build a scaled model made up of the same material and the same geometrical characteristics, i.e. a design using a length scaling (ratio between the length of the scaled wheel set and that of the full scale one, Figure 12)

$$
\varphi_{l}=e
$$

where $e$ is the scaling factor ( $e=1 / 4$ in this case). In order to insure the validity of Kalker's laws [29], the scaling strategy followed by INRETS has been used to scale the applied forces by a factor $\varphi_{F}=N^{s} / N=e^{2}$ in order to maintain the same stress level in the scaled contact patch, i.e. a stress scaling factor

$$
\varphi_{\sigma}=\frac{\sigma^{s}}{\sigma}=\frac{\varphi_{F}}{\varphi_{l}^{2}}=1
$$

On the other hand, according to the equations of structural dynamics, if a structure is scaled geometrically according to the ratio $e$, the same material properties $(E, \rho)$ and the natural
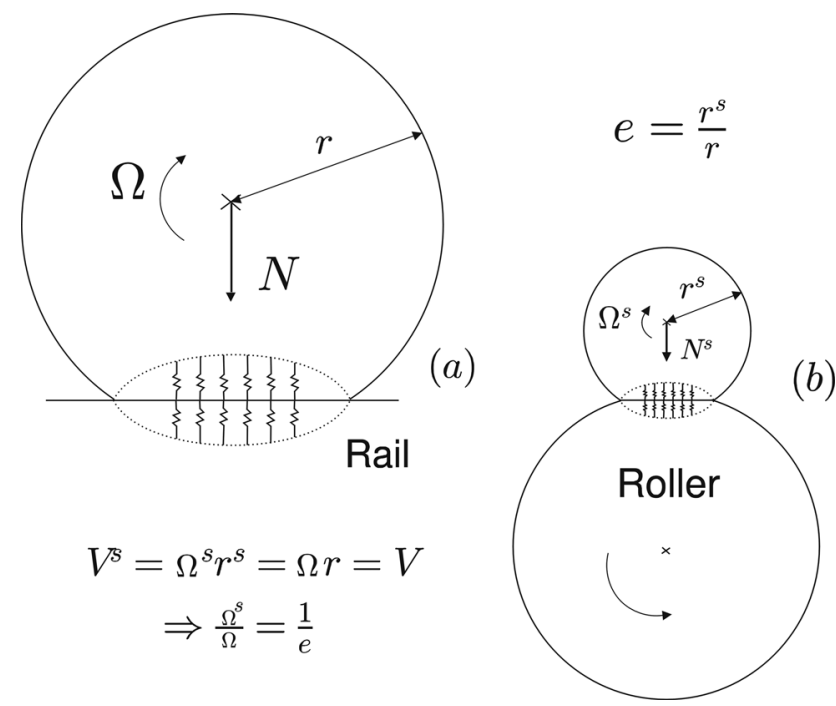

Figure 12. (a) Full-scale model of a wheel-rail contact; (b) scaled wheel-roller contact. 
frequencies are scaled according to $\omega_{n} \sim 1 / e$. If the linear speed of the scaled wheel set is the same as that of the full scale model (see Figure 12), the ratio between the scaled and full scale rotational speed is also $1 / e$. A consequence of the foregoing scaling relationships is that the full scale and the scaled system receptances $R^{s}$ and $R$ are related by the following equation:

$$
R^{s}\left(\omega^{s}\right)=\frac{1}{-\omega^{s 2} m^{s}+\mathrm{j} \omega^{s} c^{s}+k^{s}}=\frac{1}{e\left(-\omega^{2} m+\mathrm{j} \omega c+k\right)}=\frac{1}{e} R(\omega)
$$

This important property will be useful for extrapolation of wear predictions from scaled to equivalent full scale operating conditions, and is illustrated on Figure 13. A dimensional analysis gives for the other scaling coefficients

$$
\begin{aligned}
& \varphi_{\alpha}=\frac{\alpha^{s}}{\alpha}=1 \\
& \varphi_{\beta}=\frac{\beta^{s}}{\beta}=e^{2} \\
& \varphi_{k_{0}}=\frac{k_{0}^{s}}{k_{0}}=1
\end{aligned}
$$

Finally, using Equations (18), (19) and (20) into Equation (16), the relationship between the full scale and the scaled corrugation growth coefficients is insured by the following law:

$$
\begin{aligned}
G^{s}\left(\omega^{s}\right) & =-\frac{k_{0}^{s}}{2 \rho^{s} b^{s} V^{s}} \Re\left\{\frac{\alpha^{s}\left[V^{s} v_{x}^{s}-\mathrm{j} \omega^{s} F_{x}^{s} R_{x x}^{s}\right]}{\left[R_{z z}^{s}+1 / k_{c}^{s}\right]\left[1+\beta^{s} / V^{s} \mathrm{j} \omega R_{x x}^{s}\right]}\right\} \\
& =-\frac{k_{0}}{2 \rho b V} \Re\left\{\frac{\alpha\left[V v_{x}-\mathrm{j} \omega F_{x} R_{x x}\right]}{\left[R_{z z}+1 / k_{c}\right]\left[1+\beta / V \mathrm{j} \omega R_{x x}\right]}\right\}=G(\omega)
\end{aligned}
$$

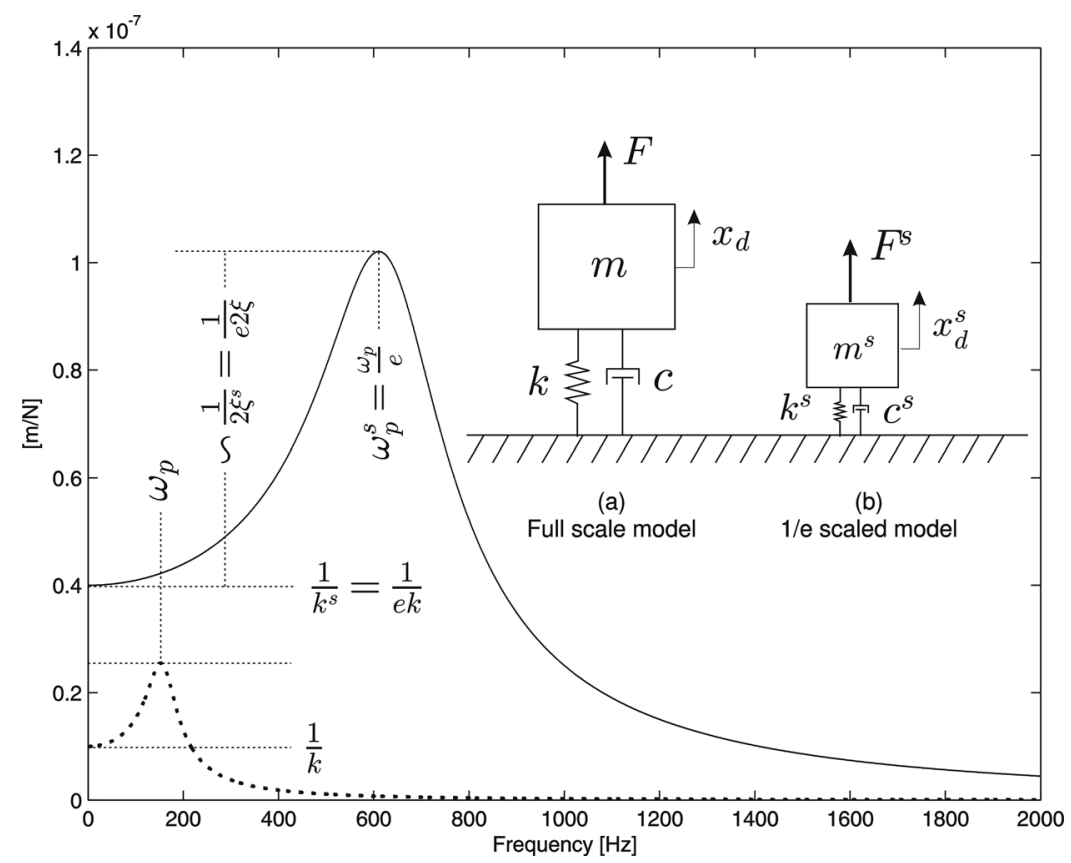

Figure 13. (a) Receptance of the full-scale model (dotted line); (b) receptance of the scaled model (solid line). 
From Equation (21), we see that the corrugation growth rate on the scaled model, $G^{s}$, equals the full scale one, $G$, for which the frequency axis has been stretched by the inverse of the scale.

\subsection{Experimental set-up}

The test bench considered in the experiment, is a quarter-scale roller rig, consisting of a scaled wheel set which is rolling on a roller, representing infinite rails (Figure 14a). The wheel set is attached to a half-bogie through geometrically scaled primary suspensions. The roller is driven by an electric motor through a transmission belt. On this bench, the lateral displacement and the yaw angle of the wheel set can be fixed independently using adjusting devices 7 and 8, respectively. Longitudinal and lateral contact forces are measured with DC force sensors 12, 13 and 11, respectively (Figure 14b). The half-bogie load is measured with a sensor (5) and the variable vertical contact force is measured using a piezoelectric force transducer connected to the shaker (sensor 15, Figure 14b). The rotational speed is measured using a tachometer (sensor 10) and the torsional vibrations are measured using a rotational

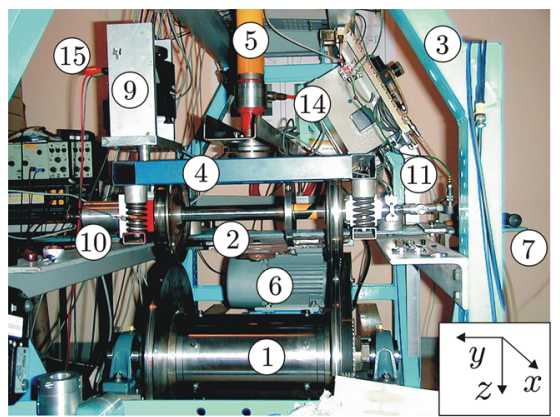

(a)

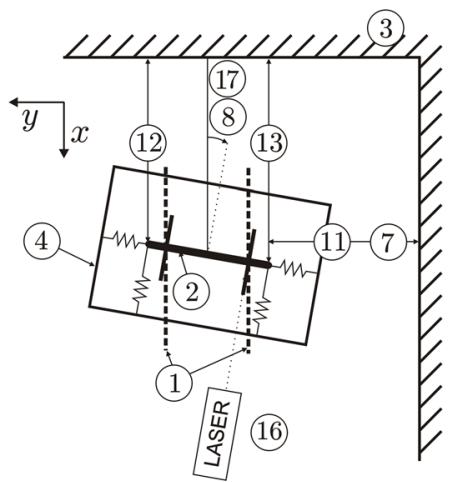

(b)

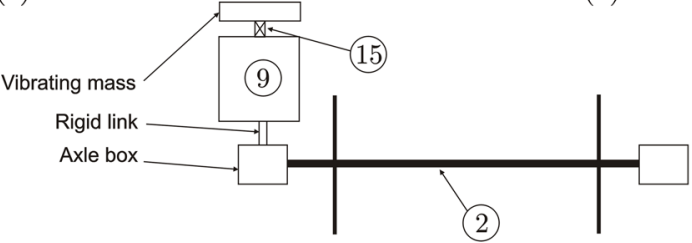

Test bench structure:

1. Roller

2. Wheel set

3. Metallic structure

4. Half bogie

Actuators:

5. Car body load

6. Driving motor

7. Lateral displacement

of the wheel set

8. Yaw angle of the wheel set

9. Shaker

\section{Sensors:}

10. Tachometer

11. Lateral force

12. Longitudinal force (right)

13. Longitudinal force (left)

14. Vertical force

15. Vertical force variation

16. Rotational laser velocimeter

17. Yaw angle of the wheel set

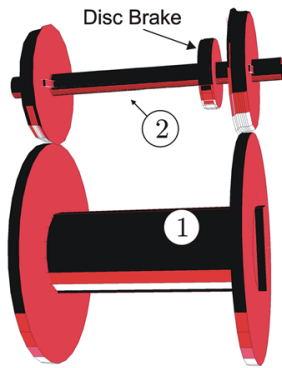

(d)

(c)

Figure 14. (a) Picture of the quarter-scaled test bench; (b) simplified drawing of a top view of the bench; (c) drawing of a front view of mounting of the shaker on the wheel set; (d) multi-body model of the test bench. 
laser Doppler vibrometer (sensor 16). Torsional vibrations can also, in principle, be measured by the tachometer, but it turns out that the signal is more noisy at high frequency. Additionally, as laser cannot distinguish between torsional and vertical vibrations of the wheel set, better results have been obtained when the laser beam points to the wheel where no variable vertical force is applied.

The wavelength-fixing mechanism described in Section 2.2 is reproduced as follows. In the curve, the outer rail is longer than the inner one. Due to the poor steering capabilities of the front bogie in curve, the difference in rolling radii is insufficient to compensate the difference in the rail length. As a result, a torsion is induced in the wheel set. On the bench, as both rails have the same length, the difference in rolling radii is imposed by a lateral displacement applied to the wheel set, resulting in a torsion of the wheel set due to the asymmetry of the wheel profiles.

The variation in the vertical force releases the torsion in the wheel set periodically, allowing roll-slip oscillations between the rail and the wheel to occur. In this experimental set-up, the rail roughness is not sufficient to generate by itself normal force variations high enough for rail corrugation to develop in a short test (see Section 2.2). For this reason, the vertical load variations are imposed by a shaker fixed directly on the (inner) axle box (actuator 9). Although the periodicity of the oscillations (i.e. the wavelength of rail corrugation) is indeed fixed at the frequency of the vertical load variations $[10,30]$, the phenomenon will be maximum when this frequency is close to a torsional resonance of the wheel set. To investigate the impact on wear of a vertical excitation at other frequencies than the resonance of the wheel set, a force with a sweep sine frequency is applied by the shaker. The contact friction variations are evaluated from the measurements of the rotational vibration of the wheels.

The experiment to reproduce the mechanism of rutting corrugation on the scaled bench has been performed in the following conditions:

- Wheel set linear constant speed: $V=5 \mathrm{~m} / \mathrm{s}$

- Lateral force: $F_{y}=700 \mathrm{~N}$

- Vertical excitation frequency: $\omega_{\text {sweep }} \in[170,250] \mathrm{Hz}$ (duration: $20 \mathrm{~s}$ )

- Vertical excitation force: $\Delta N=40 \mathrm{~N}$

\subsection{Multi-body model}

The multi-body model of the test bench (Figure 14d) has been developed in SIMPACK ${ }^{\circledR}$. The model consists of a flexible wheel set which is rolling on a rigid body roller, rolling at a constant linear speed of $5 \mathrm{~m} / \mathrm{s}$. The wheel set is represented by a finite-element model, including its first six modes (two torsional modes and four bending modes), and is linked to the inertial frame through the primary suspensions. The lateral force is achieved by a lateral displacement of the primary suspension. A sweep sine force is applied on the axle box corresponding to the non-conformal contact like depicted in Figure 3.

\subsection{Results}

Figure 15a shows the spectrogram of the longitudinal creepage simulated with the multi-body model. It shows that each frequency of the vertical force induces a variation in the rotational velocity at the same frequency. However, the longitudinal creepage is maximum when the frequency of the vertical excitation is the same as the frequency of the first torsional resonance of the wheel set. Figure 15b shows the effect of the DVA on the diagram (creepage $v s$ creep force) at the frequency of the first torsion mode. 


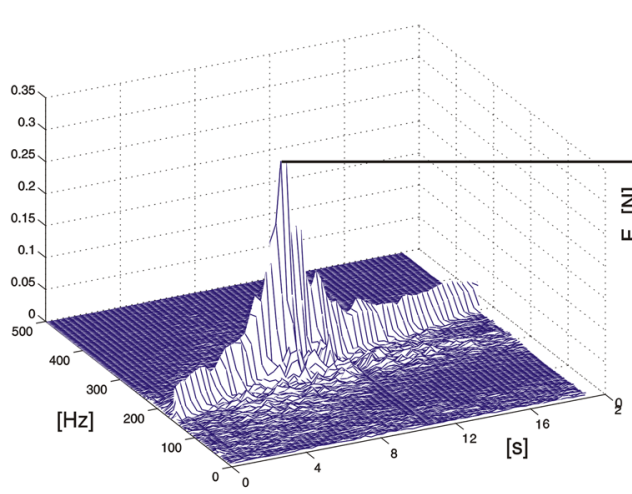

(a)

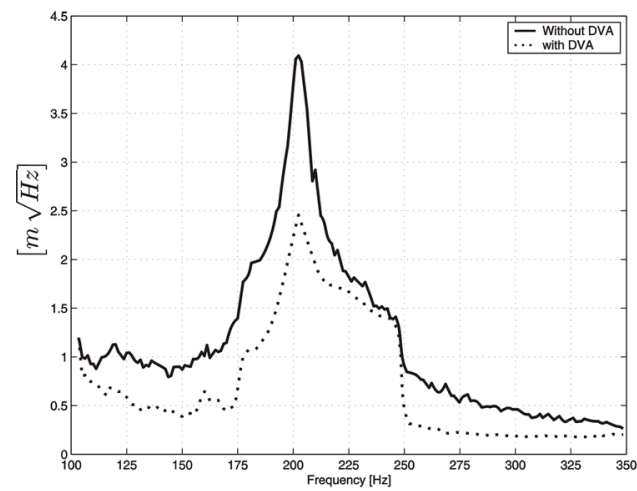

(c)

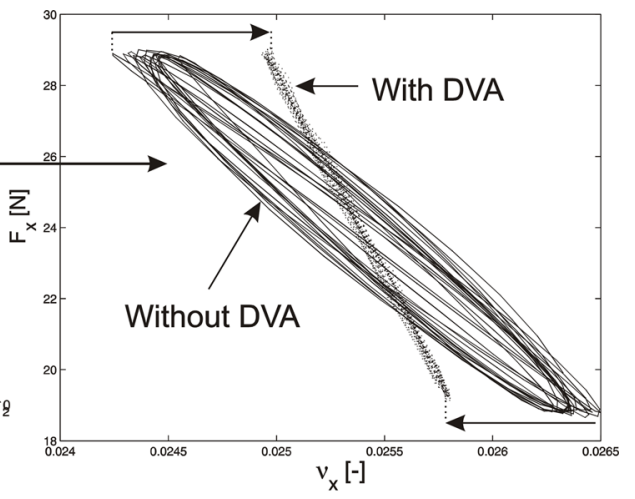

(b)

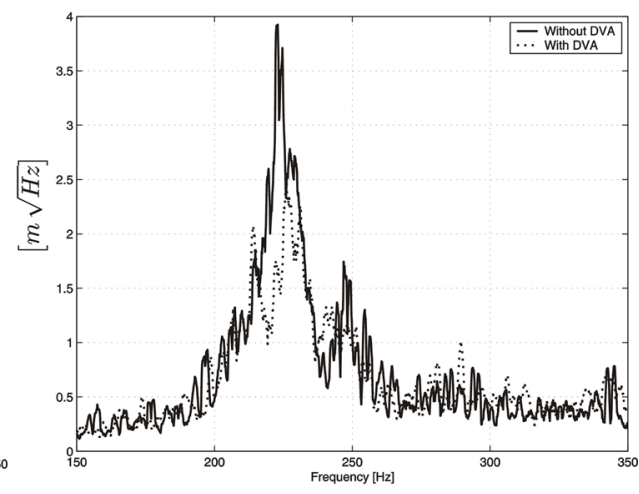

$(d)$

Figure 15. (a) Spectrogram of the longitudinal creepage without DVA; (b) effect of the DVA on the creepage creep force phase plane when the frequency of the vertical excitation corresponds to the frequency of the first torsional resonance of the wheel set; (c) fourier spectrum of the rotational velocity calculated using the multi-body model; (d) fourier spectrum of the rotational velocity measured on the bench (average on three tests).

The influence of the average longitudinal creepage has also been investigated: below the sticking limit, the area of the limit cycle in the creepage $v s$ creep force diagram increases as the average creepage increases. Above the sticking limit (as in the case shown in Figure 15b), the creepage does not affect the amplitude of the cycle any more. The DVA reduces this area by the same ratio in both cases.

The simulated and measured Fourier spectrum of the wheel rotational velocity, without and with the DVA, are compared in Figure 15c and d. In each case, the DVA has been found to reduce the peak amplitude by a factor a little less than two. The measured frequency spectrum have been averaged on three tests.

It was not possible to measure the effect of the DVA for variations in the vertical force of an amplitude significantly lower than $40 \mathrm{~N}$ because the signal-to-noise ratio was too small.

\section{Conclusions}

Rail rutting corrugation appears as a result of the combination between a torsional resonance of the wheel set and a mechanism acting to sustain these torsional vibrations, like high variations 
in the vertical contact force. The phenomenon is amplified when there is a matching between a vertical resonance of the vehicle-track system and a torsional resonance of the wheel set.

This study shows that the damping of the torsion mode of the wheel set plays a key role in the reduction of the wear process involved. A dynamic vibration absorber has been presented as a potential solution to mitigate rutting corrugation, and its design has been described. A very simple solution has been proposed, which does not require significant modifications to the existing wheel sets. Its efficiency has been demonstrated numerically and experimentally, on a quarter-scale set-up.

\section{Acknowledgements}

The authors gratefully acknowledge Hugues Chollet and the New Technologies Laboratory of INRETS for supplying the test bench and useful comments on this work. This work has been performed within the EU project Wheel-rail corrugation in urban transport - GRD2-2001-5006. The financial support of the European Union is warmly acknowledged. The comments of the reviewers have contributed to improve significantly the quality of the paper and are gratefully acknowledged. Finally we thank François Samyn (D2S International) for his contribution to the numerical study during his MSc thesis.

\section{Note}

1. Patent Pending (No. 06120344.4-2424).

\section{References}

[1] S.L. Grassie and J. Kalousek, Rail corrugation: characteristics, causes and treatments, J. Rail Rapid Transit. 207 (1993), pp. 57-68.

[2] E. Tassilly and N. Vincent, Rail corrugations: analytical model and field tests, Wear 144 (1991), pp. $163-178$.

[3] E. Tassilly and N. Vincent, A linear model for the corrugation of rails, J. Sound Vib. 150 (1991), pp. 25-45.

[4] S.L. Grassie, Rail corrugation: advances in measurement, understanding and treatment, Wear 258 (2005), pp. 1224-1234.

[5] Transit Cooperative Research Program. Report RRD-26 (Project D-01): Rail corrugation mitigation in transit, June 1998.

[6] H. Chollet, Etude En Similitude Mécanique Des Efforts Tangents Au Contact Roue-Rail, Ph.D. thesis, Université Paris 6, 1991.

[7] C.A. Brockley and P.L. Ko, An investigation of rail corrugation using friction-induced vibration theory, Wear 128 (1988), pp. 99-106.

[8] W.F. Hayes and H.G. Tucker, Wheelset-track resonance as a possible source of corrugation wear, Wear 144 (1991), pp. 211-226.

[9] A. Matsumoto, Y. Sato, M. Tanimoto, and Q. Kang, Study on the formation mechanism of rail corrugation on curved track, Vehicle Syst. Dyn. Suppl. 25 (1996), pp. 450-465.

[10] A. Matsumoto, Y. Sato, O. Hiroyuki, M. Tanimoto, Y. Oka, and E. Miyauchi, Formation mechanism and countermeasures of rail corrugation on curved track, Wear 253 (2002), pp. 178-184.

[11] S.L. Grassie and J.A. Elkins, Corrugation on North American transit lines, Vehicle Syst. Dyn. Suppl. 28 (1998), pp. 5-17.

[12] B. Ripke and K. Hempelmann, Model prediction of track loads and rail corrugation, Railway Gazette (1994), pp. 447-450.

[13] K. Hempelmann and K. Knothe, An extended linear model for the prediction of short pitch corrugation, Wear 191 (1996), pp. 161-169.

[14] J.I. Egana, J. Vinolas, and M. Seco, Investigation of the influence of rail pad stiffness on rail corrugation on a transit system, Wear 261 (2006), pp. 216-224.

[15] M. Tomeoka, N. Kabe, M. Tanimoto, E. Miyauchi, and M.Nakata, Friction control between wheel and rail by means of on-board lubrication, Wear 253 (2002), pp. 124-129.

[16] D.T. Eadie, J. Kalousek, and K.C. Chiddick, The role of high positive friction (HPF) modifier in the control of short pitch corrugations and related phenomena, Wear 253 (2002), pp. 185-192.

[17] A. Matsumoto, Y. Sato, H. Ohno, M. Tomeoka, K. Matsumoto, T. Ogino, M. Tanimoto, Y. Oka, and M. Okano, Improvement of bogie curving performance by using friction modifier to rail/wheel interface: Verification by full-scale rolling stand test, Wear 258 (2005), pp. 1201-1208.

[18] Transit Cooperative Research Programme, Wheel and rail vibration absorber testing and demonstration, Tech. Rep. 67, 2001. 
[19] J. Maes and H. Sol, A double tuned rail damper-increased damping at the two first pinned-pinned frequencies, J. Sound Vibration 267 (2003), pp. 721-737.

[20] J. Ormondroyd and J.P. Den Hartog, The theory of the damped vibration absorber, Trans. Am. Soc. Mech. Eng. 50 (1928), p. 7.

[21] S. Timoshenko and D.H. Young, Advanced Dynamics. McGraw-Hill Book, New York, 1948.

[22] Y.Z. Wang and S.H. Cheng, The optimal design of dynamic absorber in the time domain and the frequency domain, Appl. Acoust. 28 (1989), pp. 67-78.

[23] J. Connor, Introduction to Structural Motion Control, Prentice Hall, New York, 1999.

[24] Patent WO 2004/007217. Reduction of torsional vibration in rail vehicle wheel set.

[25] K. Hempelmann, F. Hiss, and B. Ripke, The formation of wear patterns on rail tread, Wear 144 (1991), pp. 179-195.

[26] J.J. Kalker, Rolling contact phenomena, in Rolling Contact Phenomena, B. Jacobs and J.J. Kalker, eds., New York, 2000.

[27] H. Krause and G. Poll, Wear of wheel-rail surfaces, Wear 113 (1986), pp. 103-122.

[28] A. Jaschinski, H. Chollet, S. Iwicki, A. Wickens, and J. Von Wurzen, The application of roller rigs to railway vehicle dynamics, Vehicle Syst. Dynam. 31 (1999), pp. 345-392.

[29] J.J. Kalker, Survey of wheel-rail contact theory, Vehicle Syst. Dynam. 5 (1979), pp. 317-358.

[30] A. Matsumoto, Y. Sato, M. Nakata, M. Tanimoto, and K. Qi, Wheel-rail contact mechanics at full scale on the test stand, Wear 191 (1996), pp. 101-106. 